premature infants. (Suk D, Krauss AN, Engel M, Perlman JM. Amplitude-integrated electroencephalography in the NICU: frequent artifacts in premature infants may limit its utility as a monitoring device. Pediatrics Feb 2009;123:e328-e332). (Respond: Jeffrey M Perlman MB ChB, Weill Cornell Medical College, Department of Pediatrics, 525 E $68^{\text {th }}$ St, Suite N-506, New York, NY 10065. E-mail: jmp2007@med.cornell.edu).

COMMENT. Amplitude-integrated EEG (aEEG) is a sensitive monitor of background cerebral activity in the early prediction of outcome after perinatal asphyxia in term infants. Abnormal aEEG is used in selection of patients for hypothermia. The above study introduces a note of caution in the use of the aEEG as an indicator of cortical function in premature infants in the NICU. The concurrent use of the conventional EEG and careful attention to electrode placement are suggested.

\title{
EVALUATION OF SLEEP DEPRIVATION IN THE PEDIATRIC EEG
}

The value of sleep deprivation to increase the yield of routine outpatient EEG in the diagnosis of epilepsy was assessed in a randomized, blinded comparison of routine EEG (REEG) versus sleep-deprived EEG (SDEEG) in 206 children aged 0 to 18 years referred by neurologists at Helen DeVos Children's Hospital, Grand Rapids, MI. Patients referred for EEG had $>1$ seizure $(83 \%)$ or unclear spells $(17 \%)$. Before the EEG, sleep-deprived patients averaged 4.9 hours sleep while those not sleep deprived had 7.9 hours sleep $(\mathrm{P}<.00001)$. Only $48 \%$ of the REEG group had adequate sleep for age vs $12 \%$ of the SD group $(\mathrm{P}<.0001)$. Of a total of 127 (64\%) patients who fell asleep during the EEG, 73\% of SDEEG group reached stage 2 sleep vs $55 \%$ of REEG group $(\mathrm{P}=.009)$. EEGs were normal in $67.7 \%$ of the REEG group vs $55.6 \%$ of SDEEG group $(\mathrm{P}=.08)$. Epileptiform discharges were recorded in $35 \%$ of REEG group vs $40 \%$ of SDEEG patients. No specific type of EEG abnormality was significantly more prevalent in the SDEEG group. Abnormal EEGs were more frequent in patients with clinically diagnosed seizures compared with those with unclear spells $(\mathrm{P}=.001)$. Odds of an abnormal EEG were also higher in the older ( $>3$ years) age group vs younger children $(\mathrm{P}=.022)$. Sleep during the EEG did not influence the proportion of patients with epileptiform records in comparison with awake EEG only. Sleep deprivation, but not sleep during the EEG, provides a modest increase in EEG epileptiform discharges in children diagnosed with seizures by neurologists. Compared with a routine EEG, 11 SDEEGs would be required to identify 1 additional child with epileptiform discharges on EEG, (DeRoos ST, Chillag KL, Keeler M, Gilbert DL. Effects of sleep deprivation on the pediatric electroencephalogram. Pediatrics Feb 2009;123:703-708). (Respond: Steven T DeRoos MD, 1300 Michigan, Suite 102, Grand Rapids, MI 49503. E-mail: steven.deroos@devoschildrens.org).

COMMENT. Routine EEG fails to confirm a diagnosis of epilepsy in approximately $50 \%$ of patients with clinical seizures. Sleep and sleep deprivation are recommended to enhance the diagnostic yield. Despite the burden on parents and child of following the protocol of sleep deprivation (Nijhof SL et al, 2005), many authorities consider the benefits of sleep-deprived EEG (SD-EEG) to outweigh the inconvenience. Some studies that support the use of SD include Rowan AJ et al (1982), Klingler D (1982), and Leach JP et al (2006) who compared the yield of EEG abnormalities in 85 patients using 3 different protocols. 